\title{
Interdisciplinary Co-design Framework in Healthcare Design for Older Adult Patients with Chronic Low-back Pain
}

\author{
Muhammad Tufail ${ }^{1}$, HaeBin Lee ${ }^{1}$, Hwang $\operatorname{Kim}^{1}$ and KwanMyung Kim ${ }^{1, *}$ \\ 1 Department of Design, Ulsan National Institiute of Science and Technology, 50 unist-gil, Ulju-gun, Eo- \\ nyang-eup, Ulsan, Republic of Korea \\ * Correspondence: kmyung@unist.ac.kr; Tel.: +82-52-217-2714
}

\begin{abstract}
This study presents three forms of interdisciplinary expertise in the healthcare design context to approach a particular multifaceted problem around the current healthcare for older adult patients with chronic low-back pain (LBP). Using an interdisciplinary co-design framework, first, our design approach performs the role of an initiator to define the problem by exploring the current context of healthcare. Second, it facilitates the experiences of experts and patients to reach the roots of the problem by functioning as a mediator. Third, our approach fulfills the primary role of healthcare design in producing new meanings considering the principles of patient-centeredness. These roles significantly contributed to the design of healthcare innovations. Our framework transformed the distributed disciplinary knowledge developed while tackling the multifaceted problem into new forms of expertise for collaboration in healthcare innovation.
\end{abstract}

Keywords: Interdisciplinary co-design; healthcare design; exercise; low-back pain; older adult patients; rehabilitation

\section{Introduction}

The traditional methods in healthcare design present limitations on tackling problems around healthcare because they are multifaceted, involving a diversity of other domain-specific expertise [1-3]. These methods aim at the exploration of experiences by social processes of identifying patient requirements. As such, healthcare designers focus on descriptive assessments of design by exploring the human experience, the context of human-design interaction, and underlines prescriptive intentions about healthcare design [4]. We present a case of an interdisciplinary co-design process in which our design approach played the role of initiator, mediator as well as its primary role of creator to address a particular problem in the healthcare context for older adult patients.

As society evolves, the problem scope expands with considerable unprecedented challenges [5]. Healthcare designers have to play their role to respond to these challenges by acquiring domain-specific expertise, such as understanding clinical needs, awareness of physiological variables and general medical literacy, and physiotherapy management to determine appropriate meanings to support the design of healthcare innovations [6]. Design researchers suggested participatory and co-design methods to deal with multifaceted problems in healthcare $[7,8]$. They typically perform their role as a facilitator to underline the capacities and activities required to effectively determine objective and productive participation [9].

In participatory design projects, however, medical professionals can only provide insights on healthcare to explain the causes of chronic diseases and suggest rehabilitation interventions. Because of their typical medical practice, such interventions typically ignore the human experience aspect [10]. Healthcare designers' role is limited to social processes of understanding user requirements to create experience and meaning for users and, thus their design practice cannot deeply explore the genuine problem in the healthcare context [11]. It posits that healthcare designers should develop alternative 
approaches to integrate the distributed disciplinary knowledge and bring domain-specific expertise in healthcare design.

There is a greater demand for healthcare innovations because of a recent significant increase in the aging population and age-related chronic conditions. Chronic low-back pain (LBP) is one of the most common chronic conditions in the aging population with a ratio of $60-85 \%$ during a person's lifespan [12-13]. The current healthcare includes physical therapy and medical care offered by the medical community, and general health services by senior care centers.

Physiotherapists and medical practitioners provide typical clinical treatment and preventive medical interventions to meet each patient's specific health requirements [14]. In most cases, patients become frustrated because of conservative treatment and continuous extensive therapies [15]. Many healthcare professionals hold stereotypical approaches, which undermine the dignity and autonomy of older adult patients [16]. Agebased discrimination has also been observed in healthcare institutes [10]. The side effects of surgery and drug remedies, patient hesitation, and funding deficits induced patients to abandon medical treatment and, therefore prefer going to senior care centers [17-19]. Senior care centers are mainly operated by medical personnel, therapists, and skilled nurses who typically offer a diverse range of exercise interventions - from aerophilic exercises to muscle-strengthening and stretching exercises [20, 21]. Patients find these interventions difficult with their weak body muscles because of intensive and maximum exertion [22], thus considered less efficient for older adults [23].

As chronic LBP accumulates in old age, patients grow concerned, leading them to implement their own set of actions to maintain health [24]. Because of inadequate literacy, the actions performed by these patients are inefficient in the management of LBP [25]. Patients face significant challenges with the current healthcare that represents the whole phenomenon as a multifaceted problem for healthcare design. Thus, the aim of the design practice should be to gain a more thorough understanding of this problem by envisioning new meanings to be applied to the current healthcare context. The key question explored in this study is, therefore: how, in practice, does a design approach lead by interdisciplinary co-design position domain-specific expertise and deploy this expertise to respond to a particular multifaceted problem?

\subsection{Trends in Healthcare Design}

Healthcare design has been extended to address fundamental and practical challenges associated with the aging population. Design for the aging population was introduced by the Royal College of Art in 1991 and followed by the Helen Hamlyn Center in 1999 to develop the theory and practice of inclusive design [26]. Design research is facilitated in the context of healthcare to positively enhance the current practices by bringing the perspectives of patients and other relevant actors to the design process.

Participatory and co-design research are deployed in healthcare to gain a deeper understanding of contextual user requirements, thereby improving the value and design process [27]. Evidence-based design (EBD), human-centered design (HCD), and research through design (RTD) are considered effective methods in the user-intervention stage to increase the potential of healthcare designs. Using these methods, designers have paid attention to acute and chronic treatment through new medical products, prosthetic replacements, and homecare designs [28]. For example, rheumatoid arthritis (RA), a chronic condition that causes disability and predominantly affects the small joint of the hands and wrist joints that cause severe pain, was targeted by Goncu-Berk and Topcuoglu [29] who designed a smart glove for patients with RA to reduce pain via electrical stimulation therapy. They report such a design practice requires background knowledge that can cover medical and physiological variables. Current care products based on treatment strategies have important limitations for older adults, such as their complexity, typical use in the hospital context, funding deficits, and care dependency when interacting with these products [4]. 
Healthcare design has also focused on preventive strategies and aims at the wellbeing, mental health, social interaction, and active living of the aging population. Mincolelli et al. [30] developed a customizable healthcare service for older adults at home care using HCD and co-design methods. They aimed to support older adult patients in place and minimize their dependency on families and caregivers. Chamberlain and Partridge [4] applied design to care products in the context of healthcare to improve the measured quality of care for frail patients admitted to hospitals. They suggested that design represents a new and progressively significant role in shifting the practice of health to allow hospital improvement teams to implement a co-design practice for quality of care. Tsekleves and Cooper [31] identify emergent trends in design for health in various dimensions, like public health, acute, chronic conditions, and older adult care. They suggest that design contributes significantly to the upcoming healthcare based on preventive care. Following the HCD approach to understanding the stigma for pelvic floor disorder in aged women, Ramírez et al. [32] designed five concept prototypes of playful types of empowering women and shifting their attention away from the disorder. Pericu [5] underlined the design process for healthcare by applying the HCD approach in the design of inclusive products and services that can potentially empower older adults in their daily life activities and encourage them for independent life.

Despite the above developments, the healthcare design still faces challenges in the design of healthcare products [33]. One of the challenges represents the lack of knowledge required for referring to the medical and physiological needs of the users under consideration. The HCD and co-design methods efficiently bridge this knowledge gap to facilitate the design space in understanding and accurately defining the given problem. However, once the problem is identified, the design direction confronts another challenge of lack of knowledge required to fulfill other domain-specific requirements beyond the design knowledge

\section{Materials and Methods}

This study was conducted in a senior care center and partially in a physiotherapy center, and an orthopedic and spine center. After gaining consent, 30 older adult patients experiencing spine-related abnormalities were recruited from a senior care center in South Korea (See Table 1 for patients' information). These patients were engaged for three days for five hours daily and their health check-ups and therapies were arranged in the presence of orthopaedists and therapists.

Table 1. Patients' information

\begin{tabular}{lcc}
\hline Study Participants & N \\
\hline Anthropometric characteristics & Total $(\mathrm{n}=30)$ \\
\hline Age range (years) & $72-83$ & \\
Weight, $\mathrm{kg}$ & 53.4 & \\
Height, cm & 151.2 & \\
Body mass index, $\mathrm{kg} / \mathrm{m} 2$ & 23.4 & \\
\hline
\end{tabular}

Given that orthopaedists, therapists, exercise trainers, and nurses accompanied the patients in medical contexts, they were considered stakeholders. We exclusively drew on the active participation of two orthopaedists, one therapist and one exercise trainer, and two nurses who had experienced older adult patients with chronic conditions and geriatric care management. Table 2 shows the fundamental information of all stakeholders included in this study. 
Table 2. Stakeholders' information

\begin{tabular}{lcc}
\hline Participants & Affiliation / Position & $\mathbf{N}$ \\
\hline Stakeholder (expert) & Total $(\mathrm{n}=6)$ \\
Orthopaedist & Orthopaedic and spine centre & 2 \\
Therapist & Physical therapy centre & 1 \\
Trainer & Exercise centre & 1 \\
Staff (Nurses) & Senior care centre & 2 \\
\hline
\end{tabular}

The research process is composed of three stages that extend the interdisciplinary codesign framework (See Figure 1).

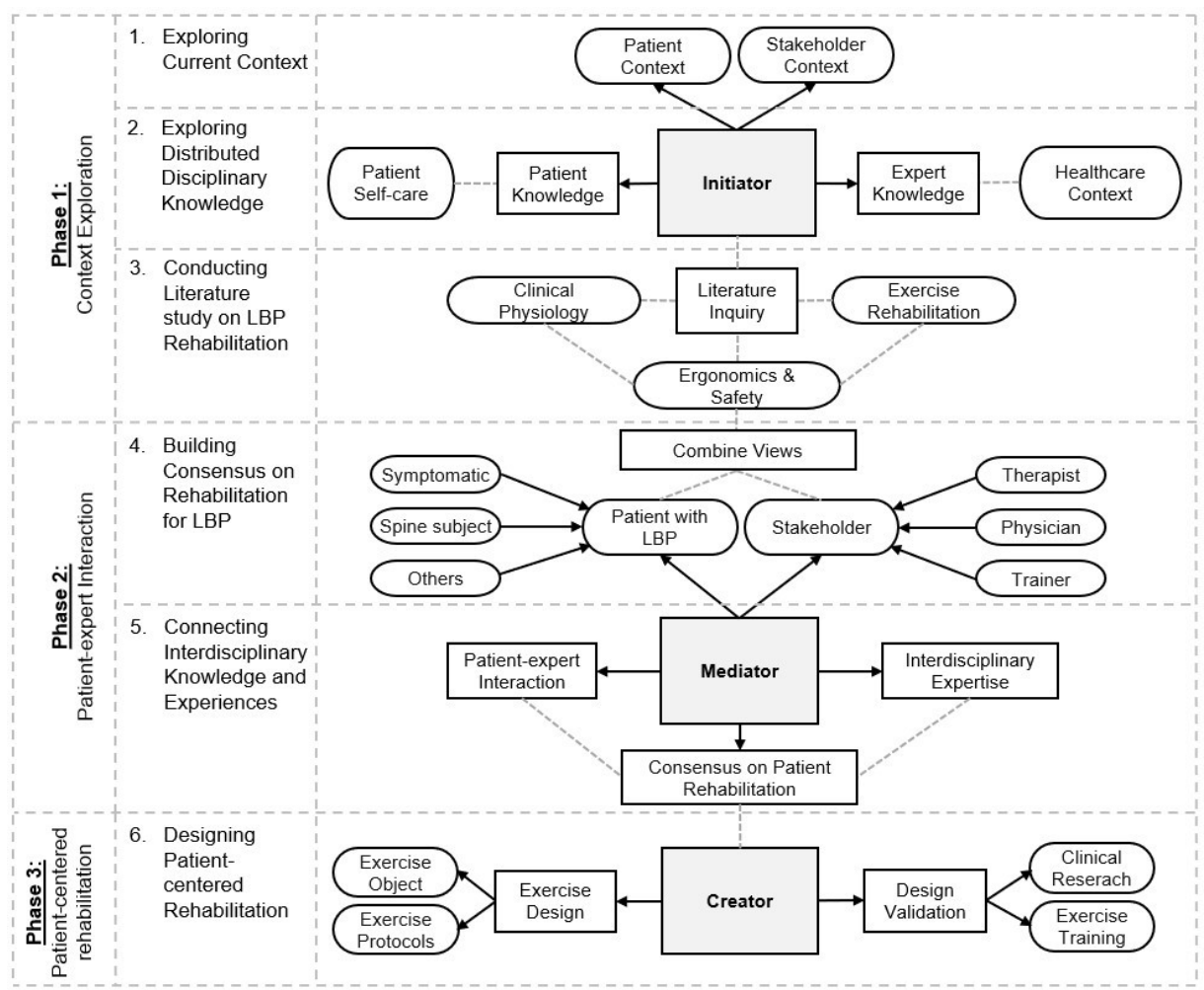

Figure 1. Interdisciplinary co-design framework

\subsection{Phase 1: Context Exploration}

\subsubsection{Exploring Current Context}

In the senior care center, patients faced LBP due to musculoskeletal conditions. They neglected proper postures during work that led to the loss of spine stability and contributed to the development of LBP. Most patients made use of electrotherapy, manual therapy, and exercise rehabilitation. Patients showed concerns, such as those caused by spine surgeries and drug remedies. They faced difficulty in adopting the existing exercises because of weakened muscle endurance causing trouble to perform strength-based exercises. Figure 2 represents the workshop activities. 


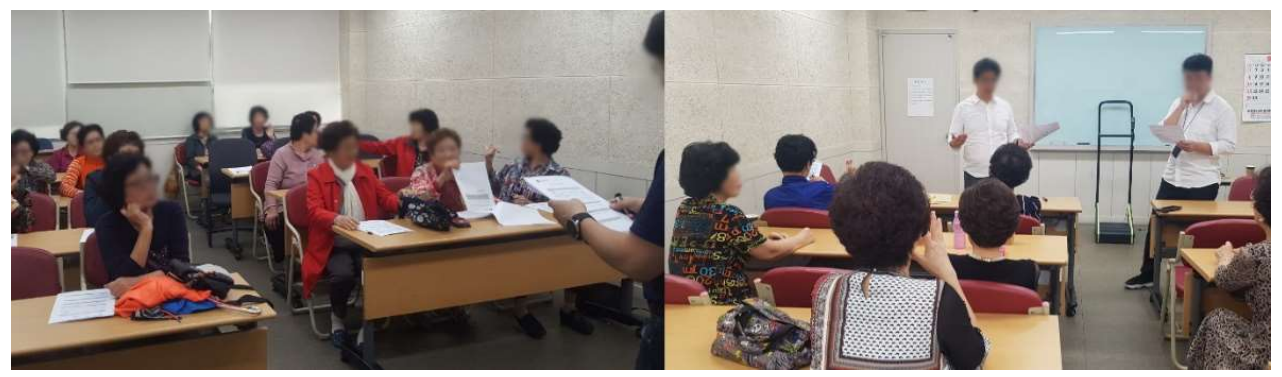

Figure 2. Workshop and open discussions with the older adults

\subsubsection{Exploring Distributed Disciplinary Knowledge}

Patients reported the side effects of conservative treatments and hardcore exercise methods, such as discomfort and tiredness. Stakeholders reported four contributing factors to LBP: spine abnormalities due to age-related conditions, prolonged sitting, poor posture stability, and back muscle stiffness. The activities of patients are shown in Figure 3.

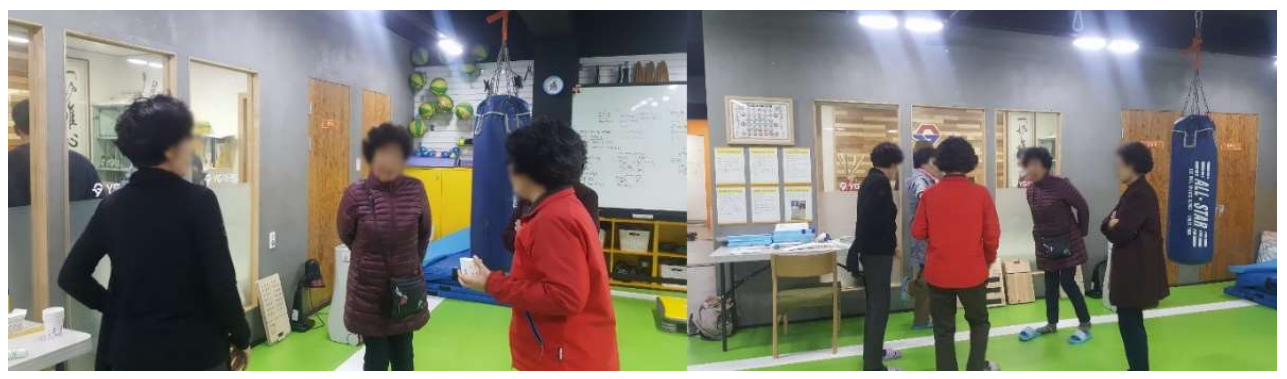

Figure 3. Patients activities in the training center

\subsubsection{Conducting a Literature Study on LBP rehabilitation}

We conducted a literature inquiry in three major fields - clinical physiology to gain medical literacy on LBP, ergonomics, and safety to identify the abilities and limitations of LBP subjects and exercise rehabilitation to acquire exercise therapy knowledge. Studies were reviewed from these fields from the years 2000 to 2021 in which older adults were the reference population and LBP symptomatic subjects. We analyzed the contents, thematized them, and clumped them to articulate mutual patterns to construct further knowledge on LBP rehabilitation (See Table 3).

Table 3. Literature inquiry matrix and mutual relationships between themes and elements

\begin{tabular}{clcccc}
\hline & & Themes in Literature & & \\
\cline { 1 - 3 } & Clinical Physiology & Ergonomics and Safety & Exercise Rehabilitation & & Mutual elements \\
\hline 1 & Muscle force & Injury recovery & Upright posture & & Muscle activation \\
2 & Muscle growth & Lateral bending & Flexibility & & Muscle endurance \\
3 & Fatigue resistance & Incline standing & Strength exercise & Trunk flexion \\
4 & Lumbar spine stability & Proper posture & Endurance posture & & Muscle contraction \\
5 & Trunk angle flexion & Static load & Squat posture & & Squat position \\
6 & Muscle activation & Standing conditions & Sports activity & & Trunk bending \\
7 & Muscle extension & Forward bending & Postural change & & Standing aid \\
8 & Trunk muscle fatigue & Partial Squatting & Flexion & & Trunk fatigue \\
9 & Voluntary contraction & Muscle fatigue & Extension & & Lateral bending \\
10 & Muscle contraction & Active movement & Endurance & & Strength \\
\hline
\end{tabular}


From the clinical physiology studies, we determined experimental studies on muscle force, muscle growth, muscle fatigue, muscle movements, and muscle contraction. In ergonomics and safety literature, we found empirical studies on muscle injury recovery, posture correction, standing conditions, and trunk muscle activities. From the exercise rehabilitation studies, we found studies on posture correction, muscle endurance exercises, standing aids, and sports activities. We also obtained invaluable insights on understanding muscle anatomy and its mechanism for our clinical research in the preceding sections.

\subsection{Phase 2: Patient-expert Interaction}

\subsubsection{Building Consensus on Rehabilitation for LBP}

All patients agreed to a medical check-up and a radiographic examination of the trunk using X-ray imaging. Among the 30 patients, 20 were observed to exhibit chronic LBP with major and minor spine deformations. The therapist and trainer manipulated customized training objects for muscle-stretching exercises. We administered interviews with the patients to recognize their views on the current training. The views of Patients A, B, and E (English translated version) are presented below. Patient A notes:

"This training is too exhaustive..., and causes discomfort and tiredness to my lower-back trunk. I feel too tired in my trunk (patient points on the thoracic vertebrae region of the spine vertebral column) and legs (the patient points to the knee and ankle joints) ..." Patient B notes:

"The exercise is helpful for my trunk muscles (patient points on the thoracic vertebrae region and lumbar vertebrae region of the spine vertebral column). I think it is much better than taking medicines or other treatment for LBP..." and Patient E notes:

"I felt mild muscle fatigue in my lower trunk in the beginning and became severe after the training (patient points to the lumbar region of the spine) ..."

Based on these reports, patients were observed by the orthopaedists to trace the areas of the body with fatigue (See Figure 4) by employing the methods from Lehman et al. [34]. These muscles were mainly Latissimus Dorsi (LD), and Lumbar Erector Spinae (LES).

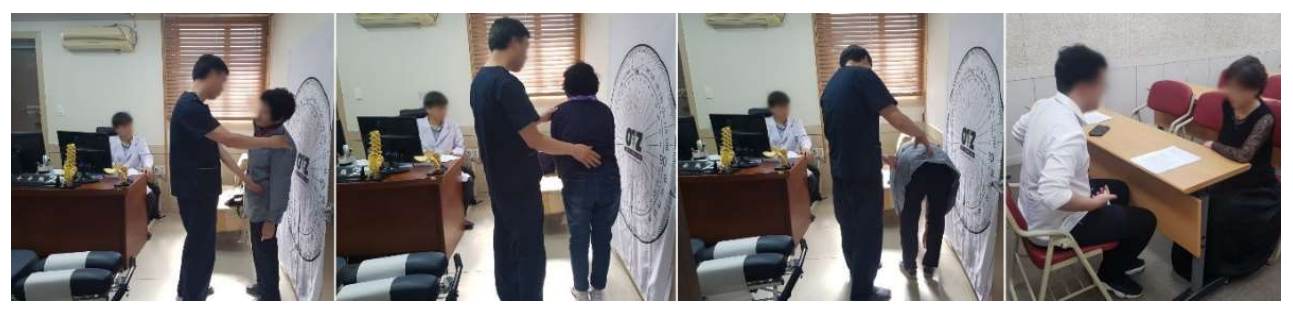

Figure 4. Patients' trunk muscles observation and medical checkup

\subsubsection{Connecting Interdisciplinary Knowledge and Experiences}

Drug intervention in LBP is common but patients cannot continue to take drug remedies because of the presence of various health problems. According to orthopaedists and the therapist, LBP was caused by prolonged sittings and sedentary activities. Trunk muscles progressively become stiffed and strained with growing age. Patients shared their concerns on the healthcare context in LBP-e.g., that it poses troubles with their weak body muscles.

We derived four practical insights. First, the patient-expert interaction endorsed a participatory co-design by exploring the knowledge and experiences. Second, the current 
practice brought all experts to the design process with uniform goals to define the current multifaceted problem. This has ultimately explored the codesign's ability to involve the actual patients and key stakeholders. Third, the interdisciplinary knowledge exchanges among the experts revealed invisible factors involved in the design process, such as awareness of physiological changes and medical requirements. Fourth, the integration of interdisciplinary knowledge and experiences among the experts led the design practice to reach an agreement on the design of exercise for trunk muscles.

\subsection{Phase 3: Patient-centred Rehabilitation}

\subsubsection{Designing patient-centered rehabilitation}

We highlighted 15 types of exercises by consulting Schilling et al. [35] and Ginn and Halaki [36] along with several exercise trials that majorly included trunk flexion and extension exercises for muscle relaxation and stabilization. In particular, these include sitting, standing, and lying down positions (plank, crunch, and bridge). The rationale behind each of these exercises was to design exercises that can manipulate LD and LES muscles. Every step of the exercise was separately presented to patients for approval.

However, the proposed exercise caused severe fatigue, which hindered consistency in the training participation. The fatigue was caused primarily by prolonged standing with the standing exercise training and its longer duration (approximately 60 minutes). Patients had difficulties in standing up from the lying down positions to perform standing exercises. Thus, eight exercise items were removed that include lying down positions, as well as those that caused severe discomfort to other parts of the body. As such, the total duration of the exercise was minimized from 60 minutes to 25 minutes, including a 7minute break. The exercise set is presented in Table 4. 
Table 4. Standing exercise set

\begin{tabular}{|c|c|c|c|}
\hline Exercise & Procedure & Anatomical position & Duration (5 sets) \\
\hline $\begin{array}{l}\text { Normal standing po- } \\
\text { sition }\end{array}$ & $\begin{array}{c}\text { Take a long breath for } \\
\text { warm-up }\end{array}$ & & $30 \mathrm{~s}$ \\
\hline \multirow{3}{*}{$\begin{array}{l}\text { Lateral bending } \\
\text { Position }\end{array}$} & $\begin{array}{l}\text { Stands with both hands } \\
\text { up }\end{array}$ & $180^{\circ}$ upward extension & $\begin{array}{l}2 \mathrm{~min} \\
\text { (Break until the next } \\
\text { item: } 1 \mathrm{~min})\end{array}$ \\
\hline & Right lateral bending & $30^{\circ}$ lateral flexion & $\begin{array}{l}3 \mathrm{~min} \\
\text { (Break until the next } \\
\text { item: } 1 \mathrm{~min} \text { ) }\end{array}$ \\
\hline & Left lateral bending & $30^{\circ}$ lateral flexion & $\begin{array}{l}3 \mathrm{~min} \\
\text { (Break until the next } \\
\text { item: } 1 \mathrm{~min} \text { ) }\end{array}$ \\
\hline \multirow{3}{*}{$\begin{array}{l}\text { Forward bending and } \\
\text { partial squat positions }\end{array}$} & $\begin{array}{l}\text { Expands the hands for- } \\
\text { ward }\end{array}$ & $90^{\circ}$ forward flexion & $\begin{array}{c}3 \mathrm{~min} \\
\text { (Break until the next } \\
\text { item: } 2 \mathrm{~min} \text { ) }\end{array}$ \\
\hline & Bends downward & $45^{\circ}$ downward flexion & $\begin{array}{l}3 \mathrm{~min} \\
\text { (Break until the next } \\
\text { item: } 2 \mathrm{~min} \text { ) }\end{array}$ \\
\hline & Bends forward slightly & $\begin{array}{l}35^{\circ} \text { eccentric squat ex- } \\
\text { tension }\end{array}$ & $3 \mathrm{~min}$ \\
\hline
\end{tabular}

There is a positive effect of standing on an incline slope surface in muscle relaxation as compared to standing on a horizontal surface [37]. The incline slope surface can create positive postural changes in both pelvic and lumbar spine angles, flexion rotation of the pelvis, and consistent improvement in lumbar spine extension. To increase the efficiency of the proposed exercise, an exercise object with $10^{\circ}, 15^{\circ}$, and $20^{\circ}$ of slope angles was developed to perform the exercise (See Figure 5) 

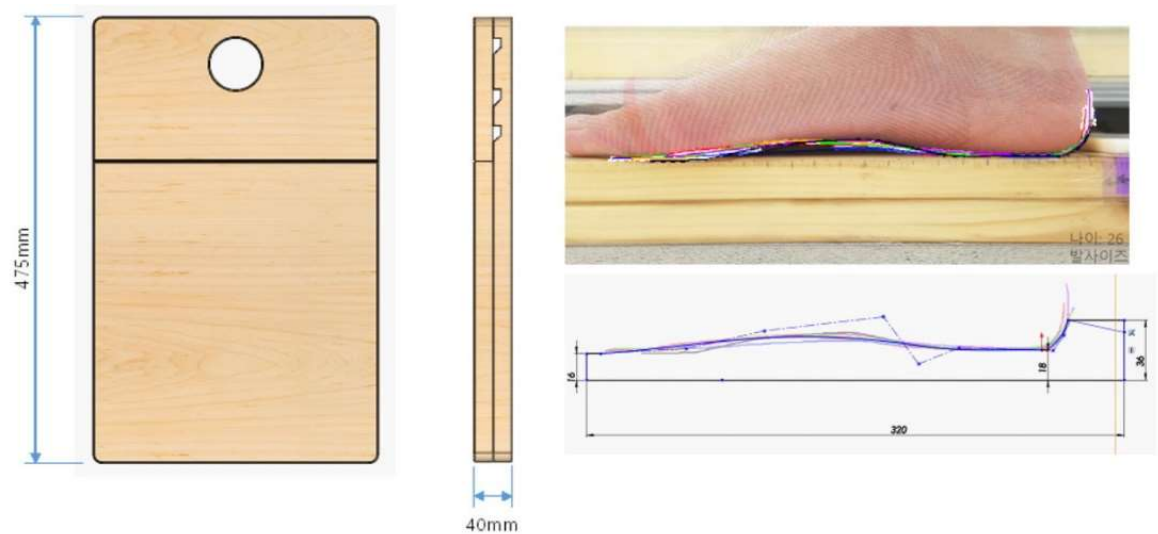

(a)
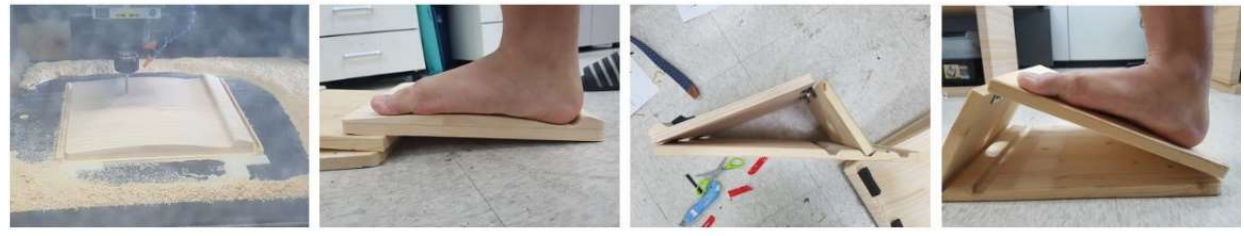

(b)
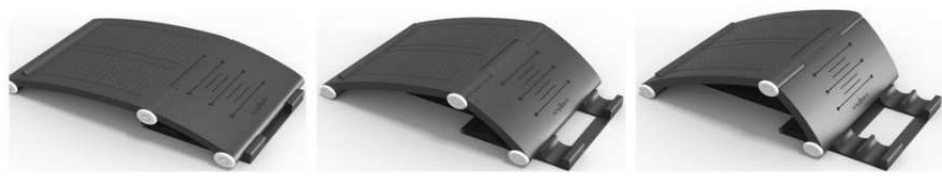

(c)

Figure 5. (a) Design idea of the incline sloped object, (b) Making prototype of the incline-sloped object, and (c) commercialized version of the exercise object

\subsubsection{Design validation for muscle activation of the trunk and LBP}

We conducted experimental trials and exercise training for five weeks based on a pretest-posttest comparison group design to test the effectiveness of the exercise object and the exercise of 16 out of the 18 patients. These patients were divided equally and randomly into control and experimental groups. The control group performed the exercise training with the level-standing position and the experimental group completed with the incline-standing position. The purpose was to measure whether there was a significant change in the activation of the LD and LES muscles and LBP over time (first week (W1), third week (W3), and fifth week (W5)) between these groups when performing the exercise. Electromyography measurements were used for muscle activation and Wong-Baker Faces Pain Rating Scale (a pain scale with 'no pain' $=0$ to 'worst pain' $=10$ ) for subjective LBP over time [38].

\section{Results}

\subsection{Muscle activation of the trunk and perceived LBP score}

Exercise training significantly influenced the activation of the LD and LES muscles and LBP at $p<0.05$. There was a significant increase in mean muscle activation and a decrease in the LBP score over time. The higher the muscle activation, the lower the LBP as the exercise training continued by the experimental group.

Table 5 depicts the LD and LES muscle activation levels of the control and experimental groups. LD and LES muscle activation levels with the normal standing position, lateral bending, forward bending, and partial squatting exercises gradually increased 
with time. The LBP score gradually decreased over time with the exercise tasks in both groups. However, the decline ratio of the LBP intensity was higher in the experimental group starting from the middle of the exercise duration, implying that enduring exercise with the incline-sloped surface object for a longer duration will relieve LBP more effectively than on a normal surface. The initial LBP score was higher than that in the subsequent weeks $(6.5 \pm 1.4)$. In the first week (W1), there was no significant difference in the LBP score between the two groups. However, there was a difference in both groups from the middle (W3) to the last week (W5). That is, in the midst (W3), the LBP score of the experimental group $(4.45 \pm 0.46)$ was lower than that of the control group $(5.2 \pm 0.5)$. In the last week (W5), the LBP score of the experimental group (3.62 \pm 0.33$)$ was lower than that of the control group $(4.41 \pm 0.5)$ at $p<0.05$. LBP score gradually decreased over time in both groups. Compared to the control group, the decline ratio of the LBP score in the experimental group was higher from the mid of the exercise training.

Table 5. Muscle activation levels

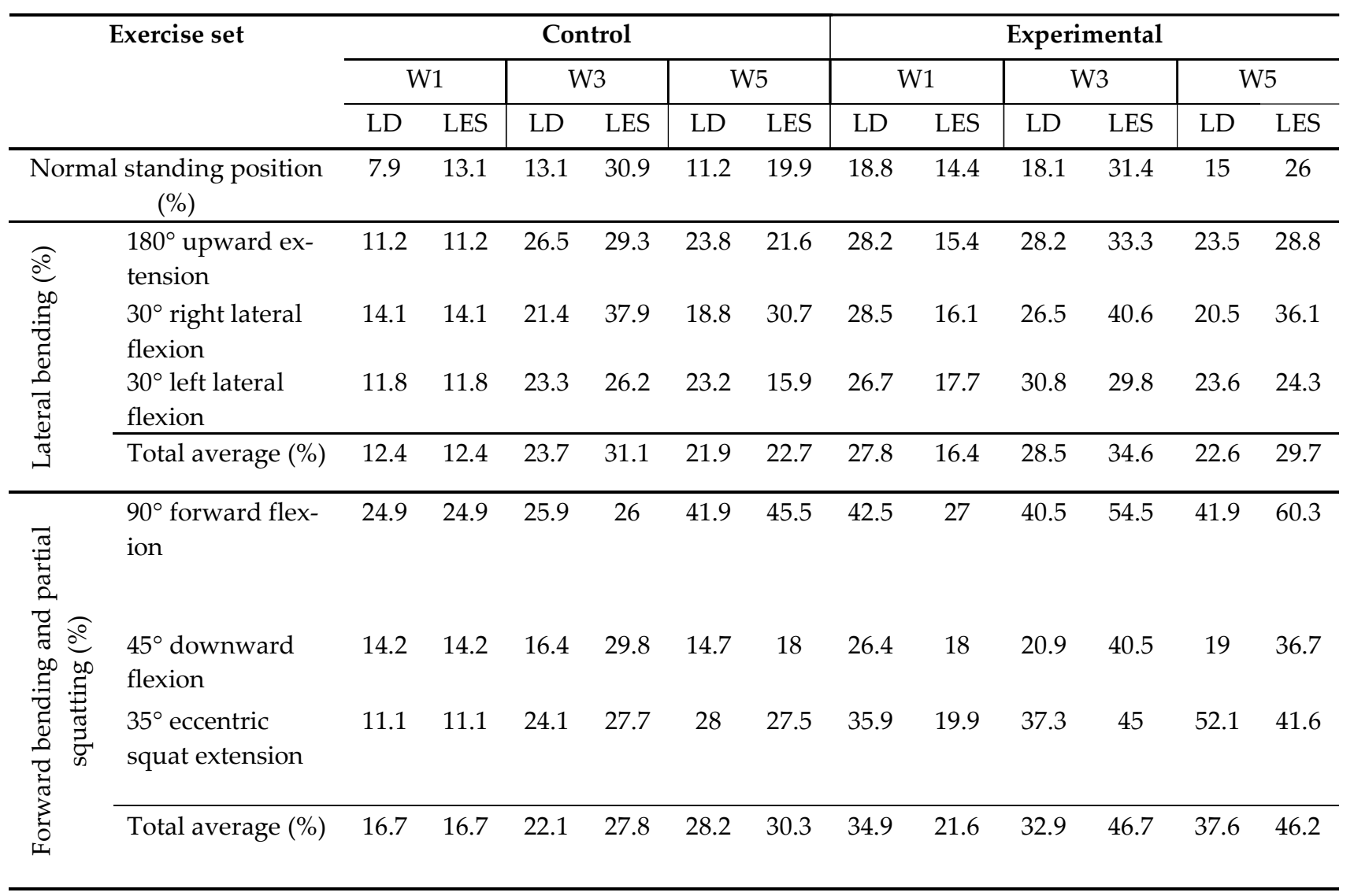

\subsection{Post-trials Interviews and Insights}

We conducted post-trial interviews with eight patients about their general experiences with exercise training. Most patients were suffered from fatigue at the start of the training. Some patients had spine surgeries because of which they possessed pain in the lower trunk. When these patients were urged for trunk exercises using the proposed exercise design, they added that exercise training was helpful and better than conservative treatments that involve complicated physiotherapeutic and clinical methods. Overall, these patients expressed favorable views on exercise training along with the exercise object. According to them, the training helped decrease the pain in their lower back. Some patients believed that training also decreased their leg pain. They furthered that the exercise object with a 10 to $15^{\circ}$ slope angle benefits muscle relaxation and posture correction. Patients requested to take the training object and the printed manual of exercise protocols 
to their homes to maintain the training in their domestic settings. These patients believed the training helped them with their knees, shoulders, and waist exercises. They, therefore, wished to suggest exercise training to their families and friends. To a large extent, we observed considerable improvements in the patients' health conditions based on the current findings.

Based on the interviews' insights, we addressed several issues that enabled the exercise object for household training purposes because most of the patients expressed interest in maintaining the exercise training. These issues were highlighted by experts and patients during the interviews. The current exercise object was made of wooden boards that needed significant changes in its shape and structure for a smooth angle adjustment, stable angle fixation, and folding function. The object in its current condition caused fatigue to the patients at the beginning of the training. Based on these grounds, we designed the object that resolves the issues. We modified its shape, material, and weight and designed angle adjustment function and balanced angle fixation mechanisms with safety features to void fall by slipping at an angle over a 15-degree slope. It was commercialized by a start-up company in late 2020.

\section{Discussion}

Participatory and co-design processes are capable to address multifaceted problems by inviting contributors with diverse disciplinary backgrounds and roles to a design team $[39,40]$. These contributors typically employ specific lenses to investigate the problems and contribute based on their own 'world' by positioning expertise in collaboration [41] and perceive the outcomes differently. Most collaborative projects are seen as difficult to commence due to the requirement of linking diverse activities of contributors, encouraging interdisciplinary negotiation, and resolving various actions and uncertainties [42]. Therefore, it entails challenges to tackle complex problems with different collaborators, especially in projects' early phases, and thus facilitating their roles, expertise, and motivation is the main difficulty, calling into question the effectiveness of the collaboration.

Acquiring interdisciplinary expertise in co-design helps immerse the design practice in the depth of the multifaceted problem and taking the role of other experts or deeply understanding their knowledge helps enhance the co-design. Healthcare designers typically rely on ethnographic approaches and employ rapid prototyping to develop co-creation with users in health reform projects. The current study defined a multifaceted problem, which consists of several challenges both in the healthcare design context and the problem itself. With the current framework, the design approach can easily be expanded and adapted to other domain-related research projects that possess characteristics of complex problems. The role of design is better purposed as initiator and mediator along with creator role to grapple with multifaceted problems.

Initiator means that the design approach gains leadership among other domain experts to define and recognize the root of the problem. A mediator means that the design approach carries unique expertise from experts into healthcare design to generate better solutions. It is challenging to transform the traditional role of design. However, designers who develop interdisciplinary expertise are required to initiate the paradigm shift of tackling multifaceted problems. The design of patient-centered rehabilitation will help other healthcare designers and researchers to embark on multidisciplinary research that challenges the traditional design practice to acquire new forms of interdisciplinary expertise in healthcare design.

The key question concerned the ability of a design approach to position domain-specific expertise in the interdisciplinary co-design and to deploy it to respond to the multifaceted problem. As the problem was multifaceted with a profound understanding of the LBP requirements, we explored the distributed disciplinary knowledge of the stakeholders by inquiring and analyzing the experts and patient knowledge. Our design approach contributed as a knowledge integrator to acquire better insights into the problem and transformed stakeholders' knowledge. The design practice needs to first initiate a problem 
exploration by engaging experts and patients regarding the problem; then lead the collaboration. Secondly, we extended the co-design to further analyze and explore the patientexpert interaction for establishing consensus among the stakeholders. Our design approach mediated the knowledge created and negotiated among the stakeholders and connected interdisciplinary knowledge and experiences that emerged during the interaction. We obtained significant contributions from this interaction by analyzing the stakeholders' knowledge and experiences to build consensus on patient rehabilitation. The mediating role in gaining this consensus thus facilitated our co-design process. The roles of initiating and leading the exploration of such a complex problem and mediating the knowledge and experiences of the stakeholders to address the problem can increase the current pace of healthcare design expansion. Once incorporated in design practice, these diverse roles will gradually inform healthcare designers with other domain knowledge and expertise over time. This will, in turn, enable them to become more credible and genuine contributors in addressing complex and multifaceted problems in a distributed disciplinary environment.

\section{Conclusions}

The interdisciplinary co-design framework could present a platform for identifying and addressing complex problems by engaging the actual patients and relevant stakeholders. Undertaking this from various perspectives, such as healthcare design, clinical physiology, ergonomics and safety, and exercise rehabilitation, the approach presents the best opportunity to effectively address emerging health concerns. The insights gained from combining these fields suggest that there are still challenges to address in enabling and supporting such multidisciplinary research. There is even such research needed to be done on developing understandings of the role of design in these fields. There are, though, multiple prospects developed from embedding design in this multidisciplinary research-e.g., providing insights into the social and behavioral challenges to foster best practices for older adults' health implementation. Assessing patients' views on challenges to lifestyle changes after their experiences in performing exercise training and responding to subsequent clinical trials in the qualitative studies will be of importance.

Design research is predominantly qualitative and mostly relies on qualitative approaches, such as participatory design, action research, and HCD, which typically lack quantitative studies. This study integrates qualitative and quantitative research outcomes that mainly emerged from the new forms of design roles as initiator and mediator to conceive the detailed design of healthcare innovations. These outcomes provided a way to approach the multifaceted problem by developing interdisciplinary expertise in design. The current problem highlighted the demand for further studies with a particular focus on interdisciplinary actions acquired by design at various phases of the design process. More studies are required to explore robustly exchangeable understandings regarding the acquired interdisciplinary expertise in healthcare design, which can address complex problems associated with the healthcare of older adult patients.

Author Contributions: Conceptualization, KwanMyung Kim. and Muhammad Tufail; methodology, Muhammad Tufail; software, Muhammad Tufail and Haebin Lee; formal analysis, Muhammad Tufail; investigation, HaeBin Lee; resources, KwangMyung Kim and Hwang Kim; data curation, Muhammad Tufail; writing - original draft preparation, KwangMyung Kim and Muhammad Tufail; writing - review and editing, KwanMyung Kim; supervision, KwanMyung Kim; project administration, KwanMyung Kim; funding acquisition, KwangMyung Kim and Hwang Kim. All authors have read and agreed to the published version of the manuscript.

Funding: This research was funded by the PROMOTION OF SPECIAL DESIGN-TECHNOLOGY CONVERGENCE GRADUATE SCHOOL OF KOREA INSTITUTE OF DESIGN PROMOTION, grant number N0001436", UNIST funded product development project, grant number 1.170097.01, and the APC was funded by KwangMyung Kim.

Institutional Review Board Statement: The study was conducted according to the guidelines of the Declaration of Helsinki, and approved by the Institutional Review Board (or Ethics Committee) of ULSAN NATIONAL INSTITUTE OF SCIENCE AND TECHNOLOGY (protocol code UNISTIRB- 
18-15-A and October 2018) the INTERNATIONAL CLINICAL RESEARCH INFORMATION SERVICE [CRIS] registration (trials registry no. KCT0004784).

Informed Consent Statement: Informed consent was obtained from all subjects involved in the study. Written informed consent has been obtained from the patient(s) to publish this paper.

Acknowledgments: The authors are thankful to the senior care center for providing subjects, space, and equipment in the experimental investigation

Conflicts of Interest: The authors declare no conflict of interest.

\section{References}

1. Dixon, D.; Brown, A.; Meenan, B.J.; Eatock, J. Experiences of new product development in the medical device industry. Medical device technology. 2006.

2. Farley, M.; Rouse, W. Technology Challenges \& Opportunities in the Biotechnology, Pharmaceutical \& Medical Device Industries. Information Knowledge Systems Management 2000.

3. Morlacchi, P.; Nelson, R.R. How medical practice evolves: Learning to treat failing hearts with an implantable device. Research Policy 2011, doi:10.1016/j.respol.2011.01.001.

4. Chamberlain, P.; Partridge, R. Co-designing co-design. Shifting the culture of practice in healthcare. Design Journal 2017, doi:10.1080/14606925.2017.1352720.

5. Pericu, S. Designing for an ageing society: products and services. Design Journal 2017, doi:10.1080/14606925.2017.1352734.

6. Lehoux, P. Medical technology into healthcare and society. A sociology of devices, innovation and governance. Sociology of Health \& Illness 2009, doi:10.1111/j.1467-9566.2009.01180_3.x.

7. de Couvreur, L.; Goossens, R. Design for (every)one: Co-creation as a bridge between universal design and rehabilitation engineering. CoDesign 2011, doi:10.1080/15710882.2011.609890.

8. Bird, M.; McGillion, M.; Chambers, E.M.; Dix, J.; Fajardo, C.J.; Gilmour, M.; Levesque, K.; Lim, A.; Mierdel, S.; Ouellette, C.; et al. A generative co-design framework for healthcare innovation: development and application of an end-user engagement framework. Research Involvement and Engagement 2021, doi:10.1186/s40900-021-00252-7.

9. Lee, Y. Design participation tactics: the challenges and new roles for designers in the co-design process. CoDesign 2008, doi:10.1080/15710880701875613.

10. Dobrowolska, B.; Jędrzejkiewicz, B.; Pilewska-Kozak, A.; Zarzycka, D.; Ślusarska, B.; Deluga, A.; Kościołek, A.; Palese, A. Age discrimination in healthcare institutions perceived by seniors and students. Nursing Ethics 2019, doi:10.1177/0969733017718392.

11. Holt, R. Design for the ages: universal design as a rehabilitation strategy. Disability \& Society 2013, doi:10.1080/09687599.2012.739364.

12. Jaul, E.; Barron, J. Age-Related Diseases and Clinical and Public Health Implications for the 85 Years Old and Over Population. Frontiers in Public Health 2017, doi:10.3389/fpubh.2017.00335.

13. Quentin C, Bagheri R, Ugbolue UC, Coudeyre E, Pélissier C, Descatha A, Menini T, Bouillon-Minois JB, Dutheil F. Effect of Home Exercise Training in Patients with Nonspecific Low-Back Pain: A Systematic Review and Meta-Analysis. International Journal of Environmental Research and Public Health. 2021,18(16):8430.

14. Pengel, L.H.M.; Refshauge, K.M.; Maher, C.G.; Nicholas, M.K.; Herbert, R.D.; McNair, P. Physiotherapist-directed exercise, advice, or both for subacute low back pain: A randomized trial. Annals of Internal Medicine 2007, doi:10.7326/0003-4819-146-11200706050-00007.

15. O' Brien, J.L.; Whitbourne, S.K. Clinical interventions to empower older women. In Women and Aging: An International, Intersectional Power Perspective; 2015 ISBN 9783319093062.

16. Lothian, K.; Philp, I. Care of older people: Maintaining the dignity and autonomy of older people in the healthcare setting. British Medical Journal 2001.

17. Piette, J.D. Editorial: Moving beyond the notion of "self" care. Chronic Illness 2010.

18. Schreiber, S.; Parent, E.C.; Moez, E.K.; Hedden, D.M.; Hill, D.; Moreau, M.J.; Lou, E.; Watkins, E.M.; Southon, S.C. The effect of Schroth exercises added to the standard of care on the quality of life and muscle endurance in adolescents with idiopathic scoliosis-an assessor and statistician blinded randomized controlled trial: "SOSORT 2015 Award Winner." Scoliosis 2015, doi:10.1186/s13013-015-0048-5.

19. Tones, M.; Moss, N.; Polly, D.W. A review of quality of life and psychosocial issues in scoliosis. Spine 2006.

20. Song, M.S.; Yoo, Y.K.; Choi, C.H.; Kim, N.C. Effects of nordic walking on body composition, muscle strength, and lipid profile in elderly women. Asian Nursing Research 2013, doi:10.1016/j.anr.2012.11.001.

21. Hayden, J.A.; Van Tulder, M.W.; Malmivaara, A. V.; Koes, B.W. Meta-analysis: Exercise therapy for nonspecific low back pain. Annals of Internal Medicine 2005.

22. Macaluso, A.; De Vito, G. Muscle strength, power and adaptations to resistance training in older people. European Journal of Applied Physiology 2004.

23. Atalay, E.; Akova, B.; Gür, H.; Sekir, U. Effect of upper-extremity strengthening exercises on the lumbar strength, disability and pain of patients with chronic low back pain: A randomized controlled study. Journal of Sports Science and Medicine 2017.

24. Bodenheimer, T.; Lorig, K.; Holman, H.; Grumbach, K. Patient self-management of chronic disease in primary care. Journal of the American Medical Association 2002. 
25. Wu, J.R.; Holmes, G.M.; Dewalt, D.A.; Macabasco-O'Connell, A.; Bibbins-Domingo, K.; Ruo, B.; Baker, D.W.; Schillinger, D.; Weinberger, M.; Broucksou, K.A.; et al. Low literacy is associated with increased risk of hospitalization and death among individuals with heart failure. Journal of General Internal Medicine 2013, doi:10.1007/s11606-013-2394-4.

26. Gieben-Gamal, E.; Matos, S. Design and Disability. Developing New Opportunities for the Design Curriculum. Design Journal 2017, doi:10.1080/14606925.2017.1352721.

27. Steen, M.; Manschot, M.; de Koning, N. Benefits of co-design in service design projects. International Journal of Design 2011.

28. Rivard, L.; Lehoux, P.; Hagemeister, N. Articulating care and responsibility in design: A study on the reasoning processes guiding health innovators' 'care-making' practices. Design Studies 2021, doi:10.1016/j.destud.2020.100986.

29. Goncu-Berk, G.; Topcuoglu, N. A Healthcare Wearable for Chronic Pain Management. Design of a Smart Glove for Rheumatoid Arthritis. Design Journal 2017, doi:10.1080/14606925.2017.1352717.

30. Mincolelli, G.; Giacobone, G.A.; Marchi, M.; Imbesi, S. New Domestic Healthcare. Co-designing Assistive Technologies for Autonomous Ageing at Home. Design Journal 2019, doi:10.1080/14606925.2019.1595435.

31. Tsekleves, E.; Cooper, R. Design for health: Challenges, opportunities, emerging trends, research methods and recommendations. In Design for Health; 2017 ISBN 9781317152507.

32. Ramirez, E.R.; Andreae, H.; Lemke, M. Addressing stigma in the design of a physical device and digital app for pelvic floor exercises. Design Journal 2019, doi:10.1080/14606925.2019.1595436.

33. Hwang, D.; Park, W. Design heuristics set for X: A design aid for assistive product concept generation. Design Studies 2018, doi:10.1016/j.destud.2018.04.003.

34. Lehman, G.J.; Buchan, D.D.; Lundy, A.; Myers, N.; Nalborczyk, A. Variations in muscle activation levels during traditional latissimus dorsi weight training exercises: An experimental study. Dynamic Medicine 2004, doi:10.1186/1476-5918-3-4.

35. Schilling, J.F.; Murphy, J.C.; Bonney, J.R.; Thich, J.L. Effect of core strength and endurance training on performance in college students: Randomized pilot study. Journal of Bodywork and Movement Therapies 2013, doi:10.1016/j.jbmt.2012.08.008.

36. Ginn, K.A.; Halaki, M. Do surface electrode recordings validly represent latissimus dorsi activation patterns during shoulder tasks? Journal of Electromyography and Kinesiology 2015, doi:10.1016/j.jelekin.2014.10.008.

37. Tufail M, Lee H, Moon YG, Kim H, Kim K. The Effect of Lumbar Erector Spinae Muscle Endurance Exercise on Perceived Lowback Pain in Older Adults. Phys Activ Rev 2021; 9(2): 82-92. Doi: 10.16926/par.2021.09.24

38. Wong, D.L.; Baker, C.M. Smiling face as anchor for pain intensity scales [2] (multiple letters). Pain 2001.

39. Wright M, Getta AD, Green AO, Kickett UC, Kickett AH, McNamara AI, McNamara UA, Newman AM, Pell AC, Penny AM, Wilkes UP. Co-Designing Health Service Evaluation Tools That Foreground First Nation Worldviews for Better Mental Health and Wellbeing Outcomes. International Journal of Environmental Research and Public Health. 2021, 18(16):8555.

40. Groot B, de Kock L, Liu Y, Dedding C, Schrijver J, Teunissen T, van Hartingsveldt M, Menderink J, Lengams Y, Lindenberg J, Abma T. The Value of Active Arts Engagement on Health and Well-Being of Older Adults: A Nation-Wide Participatory Study. International journal of environmental research and public health. 2021, 18(15):8222.

41. Kruger, C.; Cross, N. Solution driven versus problem driven design: strategies and outcomes. Design Studies 2006, doi:10.1016/j.destud.2006.01.001.

42. Sanders, E.B.-N.; Stappers, P.J. Co-creation and the new landscapes of design. CoDesign 2008, doi:10.1080/15710880701875068. 